\title{
Intralipid treatment for post operative delirium
}

\author{
Abstract \\ Postoperative delirium (POD) is a common and serious adverse event in the elderly patient \\ and is associated with significant morbidity and mortality. A new treatment for POD by \\ intravenous Intralipid injection in the recovery room is first suggested in the medical \\ literature.
}

Keywords: postoperative delirium, postoperative cognitive dysfunction, intralipid
Volume 7 Issue 5 - 2017

\author{
Joseph Eldor \\ Theoretical Medicine Institute, Israel
}

Correspondence: Joseph Eldor, Theoretical Medicine Institute, Jerusalem, Israel, Tel 02-5835528,

Email csen_international@csen.com

\section{Post operative delirium}

Delirium is defined by either the Diagnostic and Statistical Manual of Mental Disorders, Fifth Edition (DSM- 5) ${ }^{1}$ or by the 10th revision of the International Statistical Classification of Diseases and Related Health Problems (ICD 10, Table 3). ${ }^{2}$ Delirium is an acute and fluctuating alteration of mental state of reduced awareness and disturbance of attention. POD (Post Operative Delirium) often starts in the recovery room and occurs up to 5 days after surgery. ${ }^{3-5}$ One investigation ${ }^{4}$ found that many patients with POD on the peripheral ward already had POD in the recovery room.

More than 230million surgical procedures are performed each year worldwide, of which more than 80 million are in Europe..$^{6-8}$ In Europe, the in-hospital mortality rate up to a maximum of 60days is 3\% after elective surgery and nearly $10 \%$ after emergency surgery. ${ }^{7}$ In addition to mortality, postoperative cognitive impairments such as POD and postoperative cognitive dysfunction (POCD) impose a huge burden on individuals and society. ${ }^{9}$ The incidence of POD is dependent on perioperative and intraoperative risk factors. ${ }^{10}$ Therefore, the incidence of POD varies within a broad range. ${ }^{11,12}$ For example, a meta-analysis of 26 studies of POD reported an incidence of 4.0 to $53.3 \%$ in hip fracture patients and 3.6 to $28.3 \%$ in elective patients. ${ }^{13}$

Delirium is one of the most common complications following hip fracture surgery in older people. This study identified pre- and peri-operative factors associated with the development of postoperative delirium following hip fracture surgery.

Published and unpublished literature were searched to identify all evidence reporting variables on patient characteristics, on-admission, intra-operative and post-operative management assessing incident delirium in older people following hip fracture surgery. Pooled odds ratio $(\mathrm{OR})$ and mean difference of those who experienced delirium compared to those who did not were calculated for each variable. Evidence was assessed using the Downs and Black appraisal tool and interpreted using the GRADE approach.

A total of 6704 people (2090 people with post-operative delirium) from 32 studies were analyzed. There was moderate evidence of nearly a two-times greater probability of post-operative-delirium for those aged 80years and over (OR: 1.77; 95\% CI: 1.09, 2.87), whether patients lived in a care institution pre-admission (OR: 2.65; 95\% CI: $1.79,3.92$ ), and a six-times greater probability of developing post-operative delirium with a pre-admission diagnosis of dementia (OR: 6.07, 95\% CI: 4.84, 7.62). There was no association with intraoperative variables and probability of delirium.

Clinicians treating people with a hip fracture should be vigilant towards post-operative-delirium if their patients are older, have pre- existing cognitive impairment and poorer overall general health. This is also the case for those who experience post-operative complications such as pneumonia or a urinary tract infection. ${ }^{14}$

Post-operative cerebral dysfunction includes delirium, usually occurring early and reversible, and post-operative cognitive disorders, usually occurring later and prolonged. This is a frequent complication in patients older than 75years old. The two neurological pictures are often inter-related. The pathophysiology of both entities is similar and related to post-operative neuro-inflammation; therefore onset may occur independently of any surgical complication. Post-operative cerebral dysfunction is a serious organic complication. Reduction of inflammation represents the most logical preventive measure but currently there are no studies that show this to be effective. Prevention therefore means combining several minor measures, elements that fit well into programs of enhanced post-operative recovery after surgery. Diminished pre-operative cognitive status being a major risk factor, pre-operative rehabilitation combining nutritional, physical and cognitive support can be helpful. ${ }^{15}$

Postoperative delirium is a common and serious adverse event in the elderly patient and is associated with significant morbidity and mortality. It is of great importance to identify patients at risk for delirium, in order to focus preventive strategies. The aim of this article is to systematically review current available literature on preoperative risk factors for delirium after vascular surgery.

A systematic literature search was conducted using PubMed and EMBASE, using the MeSH terms and key words "delirium"“surgery" and "risk factor". Studies were retained for review after meeting strict inclusion criteria that included only prospective studies evaluating risk factors for delirium in patients who had elective vascular surgery. Diagnosis of delirium needed to be confirmed using the Diagnostic and Statistical Manual of Mental Disorders (DSM) or ICD-10.

Fifteen articles were selected for inclusion, incidence of delirium across the studies ranged from $5 \%$ to $39 \%$. Many factors have been associated with increased risk of delirium, including age, cognitive impairment, comorbidity, depression, smoking, alcohol, visual and hearing impairment, ASA-score, biochemical abnormalities, operative strategies and blood loss.

Delirium is a common complication after elective vascular surgery in elderly. The highest delirium incidence was observed after open aortic surgery as well as after surgery for critical limb ischemia. A picture starts to form of which predisposing factors lead to increased risk of delirium. The leading risk factors consistently identified in this systematic review were advanced age and cognitive impairment. Multi-disciplinary specialist-led interventions in the preoperative 
phase could decrease incidence and severity of delirium and should be focused on identified highrisk patients. ${ }^{16}$

This study ${ }^{17}$ investigates the relationship between cognitive dysfunction or delirium detected in the early postsurgical phase and the 1-year mortality among 514 hip fracture hospitalized older persons. Patients with early cognitive dysfunction or delirium experienced a 2 -fold increased mortality risk. Early post-operative cognitive dysfunction and delirium are negative prognostic factors for mortality. Premorbid cognitive impairment and dementia in older individuals negatively affect functional recovery after hip fracture. Additionally, postoperative delirium is an established risk factor for negative outcomes among hip fracture patients. While the majority of hip fracture patients experience minor post-surgical cognitive dysfunction, the prognostic value of this phenomenon is unknown. Therefore, we investigated the relationship between minor cognitive dysfunction or delirium detected in the early postsurgical phase and the 1-year mortality after index hip fracture.

We enrolled 514 patients with hip fracture (77.4\% women), aged 65years or older (mean age $83.1 \pm 7.3$ years), who underwent surgical hip fracture repair. Patients were assessed daily from the second to the fourth post-operative day and at 3, 6, and 12 months thereafter. All participants underwent comprehensive assessment, including detection of delirium by using the confusion assessment method and evaluation of cognitive function by using mini-mental state examination (MMSE; score range 0 to 30, with lower scores indicating poorer performance). In the absence of delirium, postsurgical cognitive dysfunction was defined as having low performance on MMSE. Vital status of 1year after the index fracture and date of death were gathered from local registries.

The observed 1-year mortality rate was $14.8 \%$. Men were more likely to die than women within 1year of the index fracture $(\mathrm{p}<$ 0.01). Compared to participants with better cognitive performance, those with MMSE $<24$, as well as those with delirium in the postoperative phase, showed a significantly higher 1-year mortality rate ( 23.3 versus 17.9 and $8.1 \%$, respectively). Independent of age and sex, post-operative cognitive dysfunction as well as delirium was both associated with a 2-fold increased mortality risk.

The presence of minor cognitive dysfunction in the early postsurgical phase is a negative prognostic factor for mortality among elderly hip fracture patients. The burden of minor cognitive dysfunction is likely superimposed on that of delirium in subgroups of frail patients. ${ }^{17}$

Perioperative cerebral hypoperfusion/ischemia is a major inciting factor of post-operative-delirium, which is coupled with adverse outcome in elderly patients. Cerebral oximetry enables noninvasive assessment of the regional cerebral oxygen saturation $\mathrm{rSO} 2$. This study aimed to investigate whether peri operative $\mathrm{rSO} 2$ variations were linked to delirium in elderly patients after spinal surgery.

Postoperative delirium was assessed for $48 \mathrm{~h}$ post-operative in 109 patients aged over 60years without a prior history of cerebrovascular or psychiatric diseases by the Confusion Assessment Method for the intensive care unit and the intensive care delirium screening checklist. The rSO2 values immediately before and throughout surgery were acquired. The preoperative cognitive functions, patient characteristics, and perioperative data were recorded.

During the $48-\mathrm{h}$ postoperative period, 9 patients $(8 \%)$ exhibited delirium. The patients with delirium showed similar peri operative $\mathrm{rSO} 2$ values as those without, in terms of the median lowest
rSO2 values ( $55 \%$ vs. $56 \% ; \mathrm{P}=0.876)$ and incidence $(22 \%$, both) and duration of decline of $\mathrm{rSO} 2<80 \%$ of the baseline values. The serially assessed hemodynamic variables, hematocrit levels, and blood gas analysis variables were also similar between the groups, except for the number of hypotensive events per patient, which was higher in the patients with delirium than in those without (4, interquartile range [IQR] 3 to 6 vs. 2, IQR: 1 to 3; $\mathrm{P}=0.014)$. The degree and duration of decrease of the peri operative rSO2 measurements were not associated with delirium in elderly patients after spinal surgery. ${ }^{18}$

Three-dimensional Arterial Spin Labeling (ASL) MRI was performed before surgery in a cohort of 146 prospectively enrolled subjects $\geq 70$ years old scheduled to undergo elective surgery. We investigated the prospective association between ASLderived measures of cerebral blood flow (CBF) before surgery with postoperative delirium incidence and severity using whole-brain and globally normalized voxel-wise analysis. We also investigated the cross-sectional association of CBF with patients' baseline performance on specific neuropsychological tests, and with a composite general cognitive performance measure (GCP). Out of 146 subjects, 32 (22\%) developed delirium. We found no significant association between global and voxel-wise CBF with delirium incidence or severity. We found the most significant positive associations between CBF of the posterior cingulate and precuneus and the Hopkins Verbal Learning Test-Revised total score, Visual Search and Attention Test (VSAT) score and the GCP composite. VSAT score was also strongly associated with right parietal lobe CBF. ASL can be employed in a large, wellcharacterized older cohort to examine associations between $\mathrm{CBF}$ and age-related cognitive performance. Although ASL CBF measures in regions previously associated with preclinical Alzheimer's Disease were correlated with cognition, they were not found to be indicators of baseline pathology that may increase risk for delirium. ${ }^{19}$

Oxidative stress may be involved in occurrence of postoperative delirium (POD) and cognitive dysfunction (POCD). 8 -iso-Prostaglandin F2 $\alpha$ (8-iso-PGF2 $\alpha$ ), an isoprostane derived from arachidonic acid via lipid peroxidation, is considered a gold standard for measuring oxidative stress. The present study aimed to investigate the ability of postoperative plasma 8 -iso-PGF $2 \alpha$ levels to predict POD and POCD in elderly patients undergoing hip fracture surgery.

Postoperative plasma 8-iso-PGF2 $\alpha$ levels of 182 patients were measured by an enzyme-linked immunosorbent assay. We assessed the relationships between plasma 8-iso-PGF2 $\alpha$ levels and the risk of POD and POCD using a multivariate analysis.

Plasma 8-iso-PGF2 $\alpha$ levels and age were identified as the independent predictors for POD and POCD. Based on areas under receiver operating characteristic curve, the predictive values of 8 -isoPGF $2 \alpha$ were obviously higher than those of age for POD and POCD. In a combined logistic-regression model, 8 -iso-PGF $2 \alpha$ significantly enhanced the areas under curve of age for prediction of POD and POCD. Postoperative plasma 8-iso-PGF2 $\alpha$ levels may have the potential to predict POD and POCD in elder patients undergoing hip fracture surgery. ${ }^{20}$

Risk factors for delirium following cardiac surgery are incompletely understood. The aim of this study was to investigate whether intraoperative pathophysiological alterations and therapeutic interventions influence the risk of post-operative delirium.

This retrospective cohort study was performed in a 12-bed cardio-surgical intensive care unit (ICU) of a university hospital and included patients consecutively admitted after cardiac surgery during a 2-month period. The diagnosis of delirium was made clinically 
using validated scores. Comparisons between patients with and without delirium were performed with non-parametric tests. Logistic regression was applied to identify independent risk factors. Results are given as number (percent) or median (range)

Of the 194 consecutive post-cardiac surgery patients, $50(26 \%)$ developed delirium during their ICU stay. Univariate analysis revealed that significant differences between patients with and without delirium occurred in the following intra-operative variables: duration of cardiopulmonary bypass (184 [72-299] vs. 113 (37-717) minutes, $\mathrm{p}<0.001)$, lowest mean arterial pressure (50 [30-70] vs. 55 [30-75] $\mathrm{mmHg}, \mathrm{p}=0.004)$, lowest haemoglobin level (85 [56-133] vs. $98[53-150] \mathrm{g} / \mathrm{L}, \mathrm{p}=0.005)$, lowest body temperature (34.5 [24.4-37.2] vs. [35.1 23.9-37.2] $\left.{ }^{\circ} \mathrm{C}, \mathrm{p}=0.035\right)$, highest noradrenaline support $(0.11[0.00-0.69]$ vs. $0.07[0.00-0.42] \mu \mathrm{g} / \mathrm{kg} /$ minute, $\mathrm{p}=0.001)$, and frequency of red blood cell transfusions (18 [36 \%] vs. 26 [18\%], $\mathrm{p}=0.018)$ and platelet transfusions $(23[46 \%]$ vs. $24[17 \%], \mathrm{p}<0.001)$. Only platelet transfusions remained an independent risk factor in the multivariate analysis $(\mathrm{p}<0.001)$.

In patients undergoing cardiac surgery, various intraoperative events, such as transfusion of platelets, were risk factors for the development of a post-operative delirium in the ICU. Further research is needed to unravel the underlying mechanisms. ${ }^{21}$

In this study, ${ }^{22}$ aimed to determine the risk factors and the incidence of delirium in patients who were followed postoperatively in our surgical intensive care unit for $24 \mathrm{~h}$ using the confusion assessment method (CAM)

After obtaining approval from the ethics committee, 250 patients were included in the study. Patients who were operated under general anaesthesia or regional anaesthesia and followed in the surgical intensive care unit were evaluated by the Ramsay Sedation Scale on the first postoperative day. CAM was applied to the patients who had a Ramsey Sedation Score of $\leq 4$. Patients' age, gender, American Society of Anesthesiologists (ASA) scores, preoperative risk factors, type of anaesthesia, operation time, intra-operative procedures, pain scores evaluated by the visual analogue scale (VAS) and postoperative analgesia methods were recorded.

The incidence of delirium was found to be $18.4 \%$. The average age of patients who developed delirium was greater than the others $(68.8 \pm 12.7$ and $57.6 \pm 12, p=0.001$, respectively). It was observed that a one-unit increase in the ASA score resulted in a 3.3- fold increase in the risk of delirium. The incidence of delirium in patients undergoing regional anaesthesia was $34.6 \%$, whereas it was $16.5 \%$ in patients receiving general anaesthesia $(\mathrm{p}=0.024)$. The existence of preoperative diabetes mellitus (DM) and chronic obstructive pulmonary disease (COPD) was shown to improve the development of delirium (p\&ly;0.05). Delirium incidence was significantly higher in patients who were administered meperidine for postoperative analgesia $(\mathrm{p}=0.013)$. The VAS scores of patients who developed delirium were found to be significantly higher $(\mathrm{p}=0.006)$.

As a result, we found that older age, high ASA score, preoperative DM and COPD are important risk factors for the development of delirium. Regional anaesthesia, high postoperative pain scores and meperidine use were observed to be associated with the development of delirium. In the postoperative period, addition of CAM, a simple measurement technique, to the daily follow-up forms can provide the early recognition of delirium, which is often underdiagnosed. We think that identification and prevention of effective risk factors have the primary importance for postoperative delirium. ${ }^{22}$
Delirium after cardiac surgery is a major problem. The exact mechanisms behind delirium are not understood. Potential pathways of delirium include neurotransmitter interference, global cognitive disorder, and neuroinflammation. Several predisposing and precipitating risk factors have been identified for postoperative delirium. The development of delirium following cardiac surgery is associated with worse outcomes in the perioperative period. Multiple interventions are being explored for the prevention and treatment of delirium. Studies investigating the potential roles of biomarkers in delirium as well as pharmacological interventions to reduce the incidence and duration of delirium are necessary to mitigate this negative outcome. ${ }^{23}$

Perhaps the most frequently described mechanism of brain injury in CABG surgery is based on the recognition that microemboli are generated by the surgeon manipulating the heart and aorta, through cardiotomy suctioning, and by the cardiopulmonary bypass circuit itself. Microemboli can be detected intraoperatively as high-intensity transient signals by transcranial Doppler sonography. They have the potential to lodge in cerebral microvasculature, impairing blood supply to the brain and thus cerebral oxygenation. Several phases during cardiac surgery have been associated with increased risk of embolic showers. Aortic cannulation and clamping (during application of cardiopulmonary bypass) increase the high-intensity transient signal rate, particularly if there is extensive atheroma in the ascending aorta. ${ }^{24}$ It is not surprising, therefore, that most $(81 \%)$ microemboli are generated at the point of aortic cross-clamp release. ${ }^{25}$ Retaining the shed mediastinal blood with cardiotomy suckers provides an additional source of lipid emboli and other fragments. ${ }^{26}$

\section{Conclusion}

A new treatment for POD by intravenous Intralipid injection in the recovery room is first suggested in the medical literature.

\section{Acknowledgments}

None.

\section{Conflicts of interest}

Author declares there are no conflicts of interest.

\section{Funding}

None.

\section{References}

1. American Psychiatric Association. Diagnostic and statistical manual of mental disorders (Dsm-51). (5th edn) Amer Psychiatric Pub Inc, USA. 2013.

2. International Statistical Classification of Diseases and Related Health Problems 10th Revision.

3. Olin K, Eriksdotter Jönhagen M, Jansson A, et al. Postoperative delirium in elderly patients after major abdominal surgery. $\mathrm{Br} J$ Surg. 2005;92(12):1559-1564

4. Sharma PT, Sieber FE, Zakriya KJ, et al. Recovery room delirium predicts postoperative delirium after hip-fracture repair. Anesthesia \& Analgesia. 2005;101(4):1215-1220.

5. Radtke FM, Franck M, Schneider M, et al. Comparison of three scores to screen for delirium in the recovery room. Br J Anaesth. 2008;101(3):338-343.

6. Eurostat-Your key to European statistics. 2015. 
7. Pearse RM, Moreno RP, Bauer P, et al. Mortality after surgery in Europe: a 7 day cohort study. Lancet. 2012;380(9847):1059-1065.

8. Weiser TG, Regenbogen SE, Thompson KD, et al. An estimation of the global volume of surgery: a modelling strategy based on available data. Lancet .2008;372(9633):139-144.

9. Saczynski JS, Marcantonio ER, Quach L, et al. Cognitive trajectories after postoperative delirium. $N$ Engl J Med. 2012;367(1):30-39.

10. Multer N, Lingehall HC, Gustafson Y. Delirium after cardiac surgery: incidence and risk factors. Interact Cardiovasc Thorac Surg. 2013;17:790-796

11. Dasgupta M, Dumbrell AC. Preoperative risk assessment for delirium after noncardiac surgery: a systematic review. J Am Geriatr Soc. 2006;54(10):1578-1589.

12. Dyer CB, Ashton CM, Teasdale TA. Postoperative delirium. A review of 80 primary data-collection studies. Arch Intern Med. 1995;155(5):461-465

13. Bruce AJ, Ritchie CW, Blizard R, et al. The incidence of delirium associated with orthopedic surgery: a meta-analytic review. Int Psychogeriatr. 2007;19(2):197-214.

14. Smith TO, Cooper A, Peryer G, et al. Factors predicting incidence of post-operative delirium in older people following hip fracture surgery: a systematic review and meta-analysis. Int J Geriatr Psychiatry. 2017.

15. Benhamou D, Brouquet A. Postoperative cerebral dysfunction in the elderly: Diagnosis and prophylaxis. J Visc Surg. 2016;153(6):S27-S32.

16. Raats JW, Steunenberg SL, De Lange DC, et al. Risk factors of postoperative delirium after elective vascular surgery in the elderly: A systematic review. Int J Surg. 2016;35:1-6.

17. Ruggiero C, Bonamassa L, Pelini L, et al. Early post-surgical cognitive dysfunction is a risk factor for mortality among hip fracture hospitalized older persons. Osteoporos Int. 2017;28(2):667-675.
18. Soh S, Shim JK, Song JW, et al. Postoperative Delirium in Elderly Patients Undergoing Major Spinal Surgery: Role of Cerebral Oximetry. J Neurosurg Anesthesiol. 2016.

19. Hshieh TT, Dai W, Cavallari M, et al. Cerebral blood flow MRI in the nondemented elderly is not predictive of post-operative delirium but is correlated with cognitive performance. J Cereb Blood Flow Metab. 2016.

20. Zheng YB, Ruan GM, Fu JX, et al. Postoperative plasma 8-isoprostaglandin F2 $\alpha$ levels are associated with delirium and cognitive dysfunction in elderly patients after hip fracture surgery. Clin Chim Acta . 2016;455:149-153.

21. Rudiger A, Begdeda H, Babic D, et al. Intra-operative events during cardiac surgery are risk factors for the development of delirium in the ICU. Crit Care. 2016;20:264.

22. Bilge EÜ, Kaya M, Şenel GÖ, et al. The Incidence of Delirium at the Postoperative Intensive Care Unit in Adult Patients. Turk J Anaesthesiol Reanim. 2015;43(4):232-239.

23. O Neal JB, Shaw AD. Predicting, preventing, and identifying delirium after cardiac surgery. Perioper Med (Lond) . 2016;5:7.

24. Mackensen GB, Ti LK, Phillips Bute BG, et al. Neurologic Outcome Research Group (NORG). Cerebral embolization during cardiac surgery: impact of aortic atheroma burden. Br J Anaesth. 2003;91(5):656-661.

25. Reinsfelt B, Ricksten SE, Zetterberg H, et al. Cerebrospinal fluid markers of brain injury, inflammation, and blood-brain barrier dysfunction in cardiac surgery. Ann Thorac Surg. 2012;94(2):549-555.

26. Brooker RF, Brown WR, Moody DM, et al. Cardiotomy suction: a major source of brain lipid emboli during cardiopulmonary bypass. Ann Thorac Surg. 1998;65(6):1651-1655. 